\title{
conferenceseries.com
}

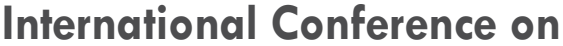 \\ Food Ghemistry \& Hydrocolloids}

August 11-12, 2016 Toronto, Canada

\section{Impact of fiber and protein derived from rapeseed press-cake on Maillard Reaction in a cookie model system}

Jonathan Wilkin, Hannah Zenker, Catherine Tsang, Antonio Dario Troise, Nikolai Zalhev and Alberto Fiore

University of Naples Federico II, Italy

R apeseed press-cake (RPC) is as a by-product of oil production, rich in proteins and fibre. Its high nutritional value opens the Rossibility to use RPC in human diet. The aim of this study was to investigate the impact of cold pressed RPC and extracted isolates enriched for fibre, polyphenols and protein, respectively, on the formation of acrylamide and 5-hydroxymethylfufural (HMF) in a cookie model system. Both Maillard reaction products (MRPs) were influenced by the ingredient: the addition of RPC as well as the protein isolate led to a significant dose-dependent increase of HMF formation in the cookie model, while acrylamide concentration was reduced up to... in presence of the fibre isolate. Three reaction mechanisms were hypothesized to be able to govern the different behaviours of acrylamide and HMF: the post-oxidative reaction of the phenolic ring with amino acids, the dicarbonyls trapping and the Michael addition of free amino acids to acrylamide. The strategy here described paved the way for the use of RPC as a tool to mitigate MRPs formation.

\section{Biography}

Jonathan Wilkin has completed his PhD in oil seeds and lipid oxidation from Cardiff Metropolitan University (2013). He Co-runs Food Innovation @ Abertay- a premier Knowledge Exchange service part of Abertay University. He is the secretary for the Institute of Food Science and Technology (IFST) Scotland Committee and is part of the Cross Party Food Group in the Scottish Government.

\section{Notes:}

